\title{
BIOMIMETIC CALCIUM CARBONATE IN THE CARBOXYMETHYL CHITOSAN /BOVINE SERUM ALBUMIN SYSTEM
}

\author{
CHENG-LI YAO, JIN-MIAO ZHU, AI-MING DING ${ }^{I^{*}}$
}

Department of Chemistry, Hefei Normal University, Hefei, Anhui,230061,China

(Received: November 10, 2011 - Accepted: July 6, 2011)

\begin{abstract}
Being a biocompatible, biodegradable and bioactive material, chitosan in the form of membranes offers a great potential as a substrate in mineralization processes in vitro. In the present work, chitosan with (without) bovine serum albumin used as templates induced the formation of calcium carbonate. The unusual morphology of calcium carbonate was get from the chitosan /bovine serum albumin system. The $\mathrm{CaCO}_{3}$ crystals obtained in system were characterized by scanning electron microscopy, Fourier transform infrared spectrography and powder X-ray diffractometry. The possible formation mechanism of $\mathrm{CaCO}_{3}$ was discussed.
\end{abstract}

Keywords: Bovine Serum Albumin, carboxymethyl Chitosan, Calcium Carbonate, Biomineralization.

\section{INTRODUCTION}

Chitosan is a partially deacetylated polymer of N-acetyl glucosamine. It is essentially a natural, water-soluble, derivative of cellulose with unique properties. Chitosan is usually prepared from chitin (2 acetamido-2-deoxy $\beta-1,4$-D-glucan) and chitin has been found in a wide range of natural sources (crustaceans, fungi, insects, annelids, molluscs, coelenterata etc. ${ }^{1}$. Specifically, it is a biocompatible, antibacterial and environmentally friendly polyelectrolyte, thus lending itself to a variety of applications ${ }^{2}$ including water treatment, chromatography, additives for cosmetics, textile treatment for antimicrobial activity $^{3}$, novel fibers for textiles, photographic papers, biodegradable films ${ }^{4}$, biomedical devices, and microcapsule implants for controlled release in drug delivery ${ }^{5-7}$.

In the shell of mollusk, the formation of nacre layers is closely related to the templating effect of organics during the crystallization of calcium carbonate; therefore, investigation into the roles of different organics involved in the crystallization is crucial in understanding biomineralization ${ }^{8}$.

Among the most current topics in this field is the adaptation of in vitro biomineralization experiments to resemble conditions in vivo more closely with two aims in mind. One aim is to gain further knowledge about basic principles of biomineralization and the second is to use this knowledge for biomimetic processing of biomaterials, with applications envisaged in bone tissue engineering especially ${ }^{9}$. Formation of $\mathrm{CaCO}_{3}$ films on the surface of chitosan membranes has been studied by some researches ${ }^{10-14}$.

We chose carboxymethyl chitosan and bovine serum albumin as matrices because they can be considered synthetic analogues of insoluble and soluble matrices in nacre, respectively. Moreover, the cooperative effects of carboxymethyl chitosan and bovine serum albumin yield aragonite thin films ${ }^{15-23}$. It has often been observed that the presence of bovine serum albumin induces the formation of aragonite crystals ${ }^{16-17}$.

This paper focuses on study of the growth of $\mathrm{CaCO}_{3}$ crystals in carboxymethyl chitosan mixed bovine serum albumin system. From the experiments we expect to find carboxymethyl chitosan -bovine serum albumin how to induce the formation of calcium carbonate.

\section{MATERIALS AND METHODS}

Chitosan and Bovine Serum Albumin (BSA) were obtained from Aldrich Chemical Company Inc, acetic acid (AR), anhydrous sodium carbonate (AR) and anhydrous calcium chloride was obtained from Shanghai Chemical Reagent Company. All reagents were used without further purification.

The preparation of carboxymethyl chitosan was prepared according to the previously described methods ${ }^{24}$. Chitosan was dissolved in $40-50 \%$ (w/w) $\mathrm{NaOH}$ solution, appropriate volume of isopropanol was added, and then chloroacetic acid was added slowly to the mixed solution. The $\mathrm{pH}$ value of reaction solution was adjusted to 7.0 with dilute aqueous $\mathrm{HCl}$ solution and the reaction was allowed to proceed at $60^{\circ} \mathrm{C}$. The obtained mixture was precipitated with acetone, washed with anhydrous alcohol at least three times, and the product was dried in vacuum.

The carboxymethyl chitosan used as the system for the growth of $\mathrm{CaCO}_{3}$ crystal, $8 \mathrm{~g}$ of carboxymethyl chitosan was added to $400 \mathrm{ml}$ of distilled water and stirred at room temperature for $40 \mathrm{~min}$. Then $400 \mathrm{~mL}$ carboxymethyl chitosan solution was divided into four equal portions which were labeled $\mathrm{CS}(\mathrm{A}) 、 \mathrm{CS}(\mathrm{B}) 、 \mathrm{CS}(\mathrm{C}) 、 \mathrm{CS}(\mathrm{D})$ respectively. At the same time there were 0 $\mathrm{mg} 、 80 \mathrm{mg} 、 160 \mathrm{mg} 、 320 \mathrm{mg}$ BSA sadded to the CS(A)、CS(B)、CS(C) 、 $\mathrm{CS}(\mathrm{D})$ respectively. After the addition of bovine serum albumin (BSA), the system was stirred slowly in order to make the solution uniform.

$0.8 \mathrm{~g}$ of anhydrous sodium carbonate was placed at the bottom of a large beaker $(250 \mathrm{~mL})$, and the same amount of anhydrous calcium chloride was placed at the bottom of a small beaker $(100 \mathrm{~mL})$. The small beaker was placed in the large beaker. The $\mathrm{CS}(\mathrm{A}) 、 \mathrm{CS}(\mathrm{B}) 、 \mathrm{CS}(\mathrm{C}) 、 \mathrm{CS}(\mathrm{D})$ was added to the two beakers, respectively. In the experiments carboxymethyl chitosan solution was not added to the beaker until the solution surface exceeded the inner small beaker wall by $5,6 \mathrm{~mm}$. The solution was kept at $37 \pm 0.1$ 、 ${ }^{\circ} \mathrm{C}$ for 7 days without stirring. When a large number of crystals present in the reaction vessel, the crystalline calcium carbonate was collected, vacuum-filtered and washed with distilled water and then with anhydrous ethanol. The crystals were dried in vacuum for $48 \mathrm{~h}$ and kept in a desiccator. The obtained crystalline $\mathrm{CaCO}_{3}$ was collected for the determination of SEM, FT-IR and XRD. 


\section{RESULTS AND DISCUSSION}
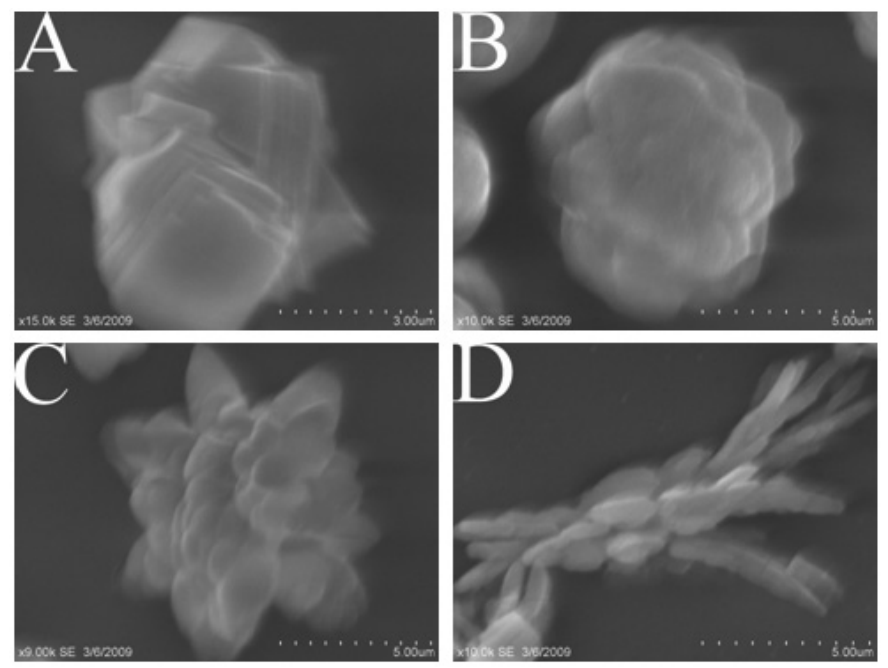

Figure 1: Morphologies of mineralized $\mathrm{CaCO}_{3}$ with different $\mathrm{BSA}$ concentrations in the carboxymethyl chitosan system( $2 \%(\mathrm{w} / \mathrm{w}))$ : (A)without BSA (B) with BSA $0.8 \mathrm{mg} / \mathrm{mL}$ (C) with BSA $1.6 \mathrm{mg} / \mathrm{mL}$ (D) with BSA $3.2 \mathrm{mg} /$ $\mathrm{mL}$.

Figure 1a d showed SEM pictures of $\mathrm{CaCO}_{3}$ particles precipitated in $2 \%$ (w/w) of carboxymethyl chitosan with different concentrations BSA. Figure1a showed there were irregular particles formed which composed of a little rhomboidal particles. With the increase of BSA concentration, cauliflowershaped crystals formed in the system (Figure1b). When the BSA concentration was up to $1.6 \mathrm{mg} / \mathrm{mL}$, symmetric dendrite-shaped $\mathrm{CaCO}_{3}$ particles were found in the system (Figure1c). Surprisingly, when BSA $=3.2 \mathrm{mg} / \mathrm{mL}$, symmetric dendrite-shaped $\mathrm{CaCO}_{3}$ particles were disappeared instead of rice ear-shaped particles (Figure1d). These results indicate that BSA had significant influence on the morphology of $\mathrm{CaCO}_{3}$ in the carboxymethyl chitosan system.

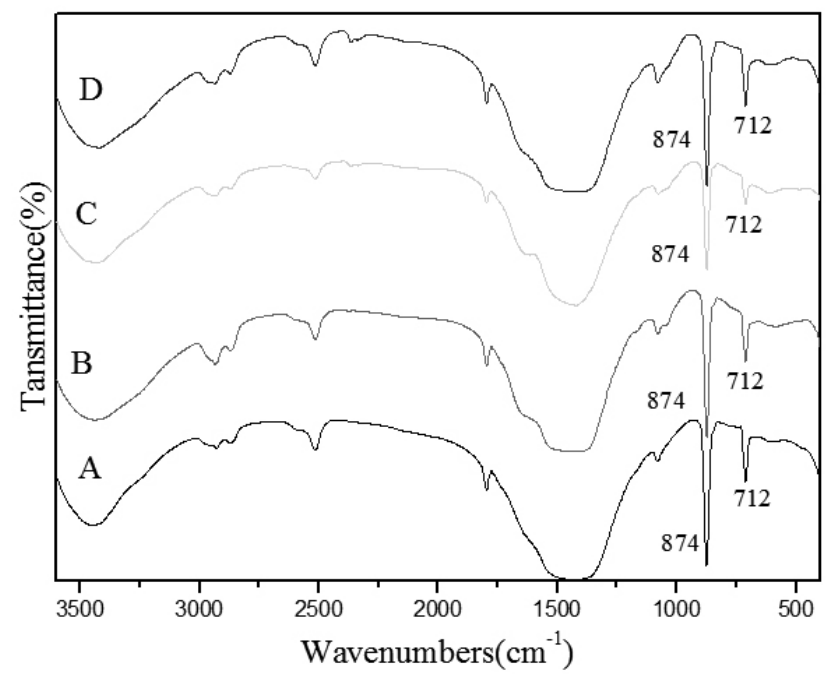

Figure 2: FT-IR spectra of $\mathrm{CaCO}_{3}$ crystals got from the carboxymethyl chitosan $(2 \%(\mathrm{w} / \mathrm{w}))$ /bovine serum albumin system: (A)without BSA (B) with BSA $0.8 \mathrm{mg} / \mathrm{mL}$ (C) with BSA $1.6 \mathrm{mg} / \mathrm{mL}$ (D) with BSA $3.2 \mathrm{mg} / \mathrm{mL}$.

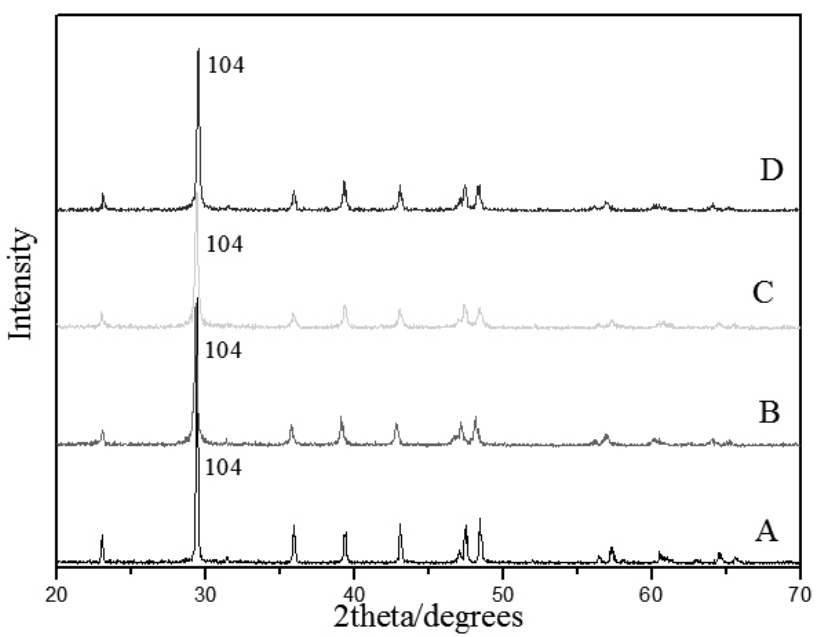

Figure 3: $\mathrm{XRD}$ patterns of $\mathrm{CaCO}_{3}$ particles obtained in the carboxymethyl chitosan $(2 \%(\mathrm{w} / \mathrm{w})) /$ bovine serum albumin system: (A)without BSA (B) with BSA $0.8 \mathrm{mg} / \mathrm{mL}$ (C) with BSA $1.6 \mathrm{mg} / \mathrm{mL}$ (D) with BSA $3.2 \mathrm{mg} / \mathrm{mL}$.

Infrared spectra of $\mathrm{CaCO}_{3}$ crystals produced in different concentration of BSA in the carboxymethyl chitosan system were shown in Figure2. While in Figure2a-d, simultaneous occurrence of absorption peaks at 874 and $712 \mathrm{~cm}^{-1}$ indicated the presence of crystalline calcite ${ }^{25}$. The slight discrepancy between experimental and published IR values could be attributed to the required grinding of a $\mathrm{CaCO}_{3}$ sample with $\mathrm{KBr}$ to produce pellets. There was little difference among those four curves. Figure 3 shows the XRD patterns of $\mathrm{CaCO}_{3}$ particles obtained in different concentrations of BSA in the chitosan systems. Using the reflection peaks at (104) plane for calcite, we can find that particles were all calcite.

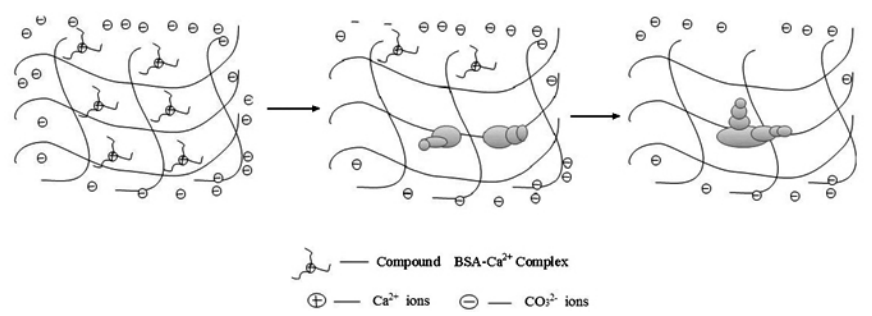

Figure 4: The possible formation mechanism of unusual morphology calcium carbonate.

(Image reprinted from Journal of the Chilean Chemical Society, 2010,55 (2): 270-273; With the authors' permission, we have done some modifications on the original picture.)

According to our previous work $26-27$, we think the carboxymethyl chitosan acts as agarose molecules in the biomineralization of calcium carbonate. Carboxymethyl chitosan molecules maybe do not directly affect the crystallization of $\mathrm{CaCO}_{3}$ which just plays a role of reaction media that control the diffusion process of ions in the media and hinder the crystal growth when the crystal size reaches the pore size of the carboxymethyl chitosan network(Figure 4).

Based on some related researches ${ }^{26-28}$, we learn some important information. The formation of $\mathrm{CaCO}_{3}$ in the carboxymethyl chitosan system with BSA should be equalized by the interplay of two parallel phenomena: First,BSA- $\mathrm{Ca}^{2+}$ polyelectrolyte composites form in the $\mathrm{CaCl}_{2}$ solution, then the electrostatic force between the $-\mathrm{COO}^{-}$and the- $\mathrm{NH}_{3}{ }^{+}$groups, and moreover, the hydrogen bond between- $\mathrm{COOH}$ and- $\mathrm{OH},-\mathrm{NH}_{2}^{3}$ groups in the network framework of carboxymethyl chitosan, will lead to the local high supersaturation with $\mathrm{CaCO}_{3}$ around the membrane when $\mathrm{CO}_{3}{ }^{2-}$ is diffused into the $\mathrm{CaCl}_{2}$ solution; Second, $\mathrm{BSA}$ remains as mobile polar groups in the form of BSA-Ca ${ }^{2+}$ in the system, and thus inhibits the growth of the crystals in the $\mathrm{CaCO}_{3}$ solution by its adsorption. During the formation of calcium carbonate, the nucleation and growth of the crystals may be affected by the carboxymethyl 
chitosan/bovine serum albumin through electrostatic matching, structural and interfacial molecular recognition. The stereo-structure of the crystal nucleus may be affected by this interfacial molecular recognition. And the activation energy of nucleation $\left(\Delta \mathrm{G}^{*}\right)$ may be decreased by interfacial molecular recognition. With the decrease of $\Delta \mathrm{G}^{*}$, the vaterite may be more easy formed and exist under this condition. The combination of these phenomena will result in a high supersaturation with $\mathrm{CaCO}_{3}$ in the carboxymethyl chitosan framework vicinity, which causes the kinetically controlled condition for crystallization and therefore leads to the formation of cauliflower-shaped crystals.

\section{CONCLUSION}

In our investigation, an easy route nucleation model is employed in the nucleation of $\mathrm{CaCO}_{3}$ under the influence of BSA in the carboxymethyl chitosan system. The results indicated that the carboxymethyl chitosan plays a role like as agarose molecules in the biomineralization of calcium carbonate. Carboxymethyl chitosan molecules just play a role of reaction media that control the diffusion process of ions in the media and hinder the crystal growth In the carboxymethyl chitosan system, bovine serum albumin will induce different shape calcium carbonate crystals but same crystal form. This research may provide new insights into the control of morphologies of $\mathrm{CaCO}_{3}$ and the controllable synthesis of novel inorganic materials.

\section{ACKNOWLEDGEMENTS}

The authors acknowledge the financial support provided by Natural Science Foundation of the Education Department of Anhui Province (No. KJ2011Z312) and key discipline of hefei normal University for carrying out this work.

\section{REFERENCES}

1. R Shepherd, S Reader ,A Falshaw, Glycoconjugate Journal, 14,535, (1997)

2. Shiro Kobayashi, Toshitsugu Kiyosada, Shin-ichiro Shoda, Journal of the American Chemical Society, 118,13113,(1996)

3. Younsook Shin, Dong Il Yoo, Kyunghye Min, Journal of Applied Polymer Science, 74,2911,(1999)

4. Shigehiro Hirano, Yasuo Koishibara, Biochemical Systematics and Ecology, 19,379, (1991)
5. A.D.Sezer,J.Akbuga, Journal of Microencapsulation, 16,687, (1999)

6. A.Bartkowiak, D.Hunkeler, Chemistry of Materials, 11,2486,(1999)

7. Takahiro Suzuki,Yasuyuki Mizushima, Tomohiro Umeda,Ryo Ohashi, Journal of Bioscience and Bioengineering, 88,194, (1999)

8. L.Addadi, D.Joester, F.Nudelman,S.Weiner, Chemistry-A European Journal, 12, 981,(2006)

9. A. L. Oliveira, J.F. Mano, R.L. Reis, Current Opinion in Solid State \& Materials Science, 7,309, (2003)

10. Linghao He, Rui Xue, Rui Song,Journal of Solid State Chemistry, 182,1082,(2009)

11. Takashi Kato, Takuo Suzuki, Takahiro Amamiya, Taku Irie, Makoto Komiyama, Supramolecular Science, 5,411, (1998)

12. Sukun Zhang, K. E. Gonsalves, Materials Science and Engineering: C, 3,117, (1995)

13. L. A. Gower, D. A. Tirrell, Journal of Crystal Growth, 191,153, (1998)

14. Fengying Zhang, Jie Wang, Zhengchi Hou, Zhengchi Hou, Ming Yu, Leidong Xie, Materials \& Design, 27,422, (2006)

15. N. Hosoda, T.Kato, Chemistry of Materials, 13, 688,(2001)

16. A. Sugawara, T. Kato, Chemical Communications, 6, 487,(2000)

17. A. Sugawara, T.Kato, Composite Interfaces, 11, 287, (2004)

18. T. Kato, Advanced Materials, 12, 1543, (2000)

19. S. Zhang, K. E.Gonsalves, Langmuir, 14, 6761, (1998)

20. S. Zhang, K. E.Gonsalves, Journal of Applied Polymer Science, 56, 687, (1995)

21. A. Kotachi, T. Miura, H. Imai, Chemistry Letters, 32,820, (2003)

22. A. Kotachi, T. Miura, H. Imai, Chemistry of Materials, 16, 3191,(2004)

23. T. Iwatsubo, K.Sumaru, T.Kanamori, T. Yamaguchi, T. Sinbo, Journal of Applied Polymer Science, 91,3627,(2004)

24. Yu-hua Shen, An-jian Xie, Xue-rong Yu, Gang Wu, Ling-guang Qiu, Shi-kuo Li, Xiang-yun Kong,Cheng-Xiang Han, Colloid Journal, 69,348, (2007)

25. John R. Clarkson, Timothy J. Price, Christopher J. Adams, Journal of the Chemical Society, Faraday Transactions, 88,243,(1992)

26. Cheng-Li Yao, Chun-Xia Qi, Jin-Miao Zhu,Wang-Hua Xu, Journal of the Chilean Chemical Society, 55,270,(2010)

27. Cheng-Li Yao, Wang-Hua Xu, Ai-Min Ding, Jin-Miao Zh, Asian Journal of Chemistry, 22, 733, (2010)

28. Wang Jinn-Mei,YAO Sony-Nian,Chinese Journal of Inorganic Chemistry, 18,249, (2002) 